\title{
Simulation de la couche limite instationnaire générée par un écoulement de houle sur un fond lisse
}

\author{
Fabrice Loiseau
}

Doctorant, Laboratoire de Mécanique de Lille - URA CNRS 1441

Bld. Paul Langevin - Cité Scientifique - 59655 Villeneuve d'Ascq

Denis Tcheukam-Toko

Doctorant, Laboratoire de Mécanique des Fluides du Havre

Quai Frissard - B.P. 265 - 76055 Le Havre Cedex

François Marin

Maitre de conférence, Laboratoire de Mécanique des Fluides du Havre

Quai F⿳亠丷厂sssard - B.P. 265 - 76055 Le Havre Cedex

\section{Résumé :}

Dans le but d'étudier le comportement de la couche limite générée par un écoulement de houle, on utilise une formulation des équations de la mécanique des fluides du type Euler-Lagrange. Ceci permet de suivre directement en lagrangien l'évolution de la surface libre.

\section{Introduction.}

Pour déterminer les écoulements côtiers, il est classique d'utiliser des données cinématiques moyennes sur la hauteur d'eau et de traiter des écoulements bidimensionnels plans horizontaux. Ceci suppose implicitement que les profils verticaux de vitesse sont semblables et nécessitent l'introduction d'une corrélation empirique (Equation de Chézy) pour évaluer le frottement sur le fond. Il est clair que les problèmes liés à la couche limite (arrachage sédimentaire, etc.) ne peuvent pas être pris en compte correctement, notamment dans le cas où la couche limite au voisinage du fond est perturbée, soit par des phénomènes prenant place sur toute la masse d'eau (la houle), soit par la structure du fond (présence de rides, etc.). Pour caractériser ces phénomènes, on se propose dans une première approche de se limiter à des écoulements bidimensionnels verticaux et de mettre en oeuvre un code en formulation A.L.E. (Arbitrary Lagrangian Eulerian) particulièrement bien adapté aux problèrnes à surface variable et nous permettant d'étudier à partir d'un domaine globale (le domaine d'écoulement) des problèmes locaux (la couche limite).

\section{Formulation initiale du problème.}

Les hypothèses choisies pour traiter le problème sont :

- écoulement bi-dimensionnel dans un plan vertical : $x-z$ 
- écoulement incompressible

- forces extérieures autres que la gravité, négligées: $\quad \vec{f}=\rho \vec{g}$ Les équations de la mécanique des fluides prennent ainsi la forme :

$$
\begin{aligned}
& \operatorname{div}(\vec{u})=0 \\
& \rho \frac{\partial \vec{u}}{\partial t}+\operatorname{div}(\rho \vec{u} \otimes \vec{u})=-\operatorname{grad}(P)+\operatorname{div}(\vec{\sigma})+\vec{f} \\
& \text { avec: } \vec{u}=\left\{\begin{array}{l}
u \\
v
\end{array}\right\} \quad \text { et } \quad \overline{\bar{\sigma}}=2 \mu \overline{\bar{D}} \quad \text { où }: \overline{\bar{D}}=\frac{1}{2}\left(\operatorname{grad}(\vec{u})+\operatorname{grad}(\overrightarrow{\mathrm{u}})^{\mathrm{t}}\right)
\end{aligned}
$$

\section{Formulation A.L.E.}

Nous utilisons une formulation faible des équations (1) et (2) : méthode des volumes finis (FVM) et une décomposition de l'opérateur temporel $\frac{d}{d t}$ en deux opérateurs distincts, l'un correspondant à une première phase dite lagrangienne (3) et l'autre à une seconde, dite eulerienne (4):

$$
\begin{aligned}
& \left\{\begin{array}{l}
\left.\cdot \frac{d}{d t}\right|_{\mid \vec{u}}\left[\int_{s} \rho d v\right]=0 \\
\left.\cdot \frac{d}{d t}\right|_{\vec{u}}\left[\int_{s}(\rho \vec{u}) d v\right]=\left(\oint_{I}((p+\vec{\sigma}) \cdot \vec{n}) d \Gamma+\int_{s} \vec{f} d v\right)
\end{array}\right. \\
& \left\{\begin{array}{l}
\left.\cdot \frac{d}{d t}\right|_{\vec{u}_{g}-\vec{u}}\left[\int_{s} \rho d v\right]=\oint_{\Gamma}\left(\rho\left(\vec{u}_{g}-\vec{u}\right) \cdot \vec{n}\right) d \Gamma \\
\left.\bullet \frac{d}{d t}\right|_{\vec{u}_{g}-\vec{u}}\left[\int_{s}(\rho \vec{u}) d v\right]=\oint_{\Gamma}\left(\rho \vec{u}\left(\vec{u}_{g}-\vec{u}\right) \cdot \vec{n}\right) d \Gamma
\end{array}\right.
\end{aligned}
$$

S est un volume fluide quelconque de frontière $\Gamma$ et $\vec{u}_{g}$ est la vitesse en tout point de la frontière $\Gamma$.

Pour discrétiser spatialement (3) et (4), nous avons opté pour une discrétisation du type éléments finis (FEM), iso-paramétrique pour la vitesse. Les éléments sont des quadrilatères. La vitesse est supposée bilinéaire sur chaque élément. Les contraintes, la masse volumique et la viscosité moléculaire y sont prises constantes.

La discrétisation des dérivées temporelles est du type différences finies avec une formulation au premier ordre en temps entre les instants $t^{\mathrm{n}}$ et $t^{\mathrm{n}+1}$.

\subsection{Etape lagrangienne.}

Dans l'équation de quantité de mouvement, les termes correspondants aux contraintes visqueuses et aux forces volumiques sont pris à l'instant $t^{n}$, les termes correspondant à la pression sont pris à l'instant $t^{n+1}$. De $t^{n}$ à $t^{n+1}$, les grandeurs 
$(\vec{u}, P)$ liées à une maille fluide, évoluent de $\left(\vec{u}^{n}, P n\right)$ à $\left(\vec{u}^{1}, P^{l}\right)$ où $\left(\vec{u}^{1}, P^{l}\right)$ sont des grandeurs définies à l'instant $t^{n+1}$ sur la maille qui s'est déplacée en suivant le fluide.

Les équations (3) se traduisent par:

$$
\left\{\begin{array}{l}
\cdot \oint_{\Gamma_{m}^{n}}\left(\vec{u}^{1} \cdot \vec{n}\right) d \Gamma=0 \\
\vec{u}^{1}-\vec{u}^{n}=\frac{\Delta t}{\tilde{\rho}_{n}^{n} \cdot S_{n}^{n}}\left[\oint_{\Gamma_{n}^{n}}\left(\left(-P^{l} \cdot \delta_{i j}+2 \mu \bar{D}^{n}\right) \cdot \vec{n}\right) d \Gamma+\int_{S_{n}^{n}} \vec{f} d v\right]
\end{array}\right.
$$

$\tilde{\rho}_{n}^{n}$ étant la masse volumique moyenne sur la surface $S_{n}^{n} d^{t} u n$ noeud de contour $\Gamma_{n}^{n}$. La décomposition de la pression suivant le schéma : $\mathrm{P}^{l}=\mathrm{P}^{n}+\Delta \mathrm{P}$ et l'introduction de la variable intermédiaire $\vec{u}^{y}$ :

$$
\vec{u}^{* 1}=\vec{u}^{n}+\frac{\Delta t}{\tilde{\rho}_{n}^{n} \cdot S_{n}^{n}}\left[\oint_{\Gamma_{n}^{n}}\left(\left(-P^{n} \cdot \delta_{i j}+2 \mu \bar{D}^{n}\right) \cdot \vec{n}\right) d \Gamma+\int_{S_{n}^{n}} \vec{f}^{n} d v\right]
$$

permettent une réécriture des équations (5) sous la forme :

$$
\left\{\begin{array}{l}
\frac{1}{\Delta t} \int_{\Gamma_{m}^{n}}\left(\vec{u}^{* 1} \cdot \vec{n}\right) d v=\oint_{\Gamma_{m}^{n}}\left(\frac{1}{\tilde{\rho}_{n}^{n} \cdot S_{n}^{n}} \oint_{\Gamma_{n}^{n}}\left(\left(\Delta P \cdot \delta_{i j}\right) \cdot \vec{n}\right) \cdot \vec{n} d \Gamma\right) d \Gamma \\
\vec{u}^{l}=\vec{u}^{* 1}+\frac{\Delta t}{\tilde{\rho}_{n} \cdot S_{n}^{n}} \oint_{\Gamma_{n}^{n}}\left(\left(-\Delta P \cdot \delta_{i j}\right) \cdot \vec{n}\right) d \Gamma
\end{array}\right.
$$

Après discrétisation spatiale, la relation (6.1) est équivalente à un système linéaire d'inconnue $\Delta \mathrm{P}_{(\mathrm{i}, \mathrm{j})}$. Une méthode d'inversion directe (LU) est utilisée pour résoudre le système d'équations. Ceci permet d'obtenir, en fin de période lagrangienne, les deux variables discrètes :

$$
\left\{\begin{array}{l}
\cdot \mathrm{p}^{\mathrm{l}}=\mathrm{P}^{\mathrm{n}}+\Delta \mathrm{P} \\
\overrightarrow{\mathrm{u}}^{\mathrm{u}}
\end{array}\right.
$$

définies sur les mailles déplacées suivant : $\quad \vec{x}^{l}=\vec{x}^{n}+\vec{u}^{1} \cdot \Delta t$

\subsection{Etape eulerienne.}

D'une manière analogue, les équations (4) se traduisent par:

$$
\left\{\begin{array}{l}
\cdot \frac{1}{\Delta t}\left[\int_{S_{n}^{n+1}} \tilde{\rho}_{n}^{n+1} d v-\int_{S_{n}^{n}} \tilde{\rho}_{n}^{n} d v\right]=\oint_{\Gamma_{n}^{n}}\left(\tilde{\rho}_{n}^{n}\left(\vec{u}_{g}-\vec{u}^{l}\right) \cdot \vec{n}\right) d \Gamma \\
\frac{1}{\Delta t}\left[\int_{S_{n}^{n+1}} \tilde{\rho}_{n}^{n+1} \vec{u}^{n+1} d v-\int_{S_{n}^{n}} \tilde{\rho}_{n}^{n} \vec{u}^{l} d v\right]=\oint_{\Gamma_{n}^{n}}\left(\vec{\rho}_{n}^{n} \vec{u}^{1}\left(\vec{u}_{g}-\vec{u}^{n}\right) \cdot \vec{n}\right) d \Gamma
\end{array}\right.
$$


Lors de la discrétisation spatiale, nous utilisons un schéma "différences amonts" pour évaluer les intégrales : $\oint_{\Gamma_{n}^{n}}\left(\tilde{\rho}_{n}^{n}\left(\vec{u}_{g}-\vec{u}^{\prime}\right) \cdot \vec{n}\right) \mathrm{d} \Gamma$ et $\oint_{\Gamma_{n}^{n}}\left(\vec{\rho}_{n}^{n} \vec{u}^{\prime}\left(\vec{u}_{g}-\vec{u}^{l}\right) \cdot \vec{n}\right) \mathrm{d} \Gamma$.

\subsection{Définition de la surface libre}

A chaque instant les noeuds de la surface libre se déplacent à la vitesse du fluide :

$$
\overrightarrow{\mathrm{x}}_{\text {surface }}^{\mathrm{n+1}}=\overrightarrow{\mathrm{x}}_{\text {surface }}^{\mathrm{n}}+\overrightarrow{\mathrm{u}}_{\text {surface }}^{1} \Delta t
$$

Pour déterminer la vitesse de grille, on impose dans un premier temps une condition supplémentaire : ne pas effectuer de déplacement suivant $x$ de la grille de calcul afin d'éviter une déformation du

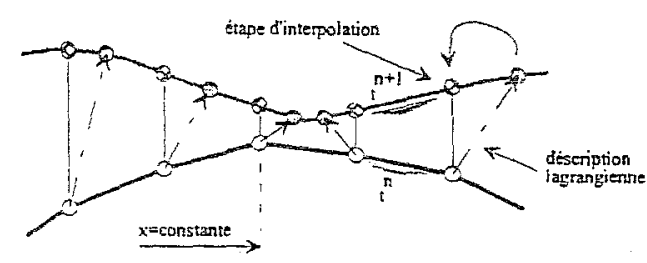

nigure 1 : déscription de l'évolution temporelle de la surface libre maillage trop importante. Il nous faut donc déterminer le déplacement de la surface libre sur des lignes de coordonnée : $x=c^{\text {te }}$. Ceci implique une phase d'interpolation. On utilise des fonctions quadratiques permettant de calculer, pour une abscisse $\mathrm{x}$ donnée, la dénivellation correspondante. Le déplacement vertical de la surface libre connue, on effectue une répartition homogène de celui-ci sur tout le domaine à l'aide d'une fonction poids $f$ :

$$
\Delta z_{(i, j)}=f_{(i, j)} \cdot \Delta z_{\text {surface }}
$$

ce qui nous amène à la formulation globale de la vitesse de grille :

$$
\left\{\begin{array}{l}
\cdot u_{g(i, j)}=0 \\
\cdot v_{g(i, j)}=f_{(i, j)} \cdot\left(\left.\frac{\Delta z}{\Delta t}\right|_{\text {surface }}\right)
\end{array}\right.
$$

\section{Caractérisation de la couche limite générée par un écoulement de houle.}

\subsection{Description du domaine d'étude.}

Le domaine de discrétisation spatiale est défini ci-dessous (figure 2), la hauteur d'eau au repos est identique à celle du canal à houle du Laboratoire de Mécanique des Fluides du Havre :

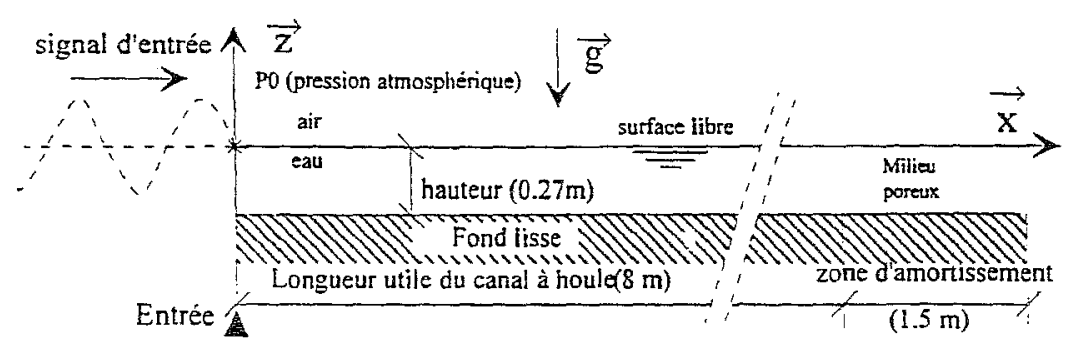

figure 2 : représentation de la zone de discrétisation spatiale 
Le maillage utilisé dans la zone d'écoulement utile est constitué de $800 * 37$ points soit un pas spatial horizontal $\Delta \mathrm{x}$ de $10^{-2} \mathrm{~m}$ pour un pas vertical $\Delta z$ variant de $4,72510^{-4} \mathrm{~m}$ à $1,8910^{-2} \mathrm{~m}$ (figure 9 ).

Les deux milieux, air et eau, sont caractérisés par leurs grandeurs spécifiques : viscosité moléculaire $\mu$ et masse volumique $\rho$.

Un modèle de turbulence du type longueur de mélange couplé avec une fonction de coupure est utilisé.

\subsection{Conditions aux limites.}

Les conditions aux limites appliquées aux frontières du domaine sont :

- aul fond :

$$
\text { - fond immobile: } \quad \vec{u}=\overrightarrow{0}
$$

- à la surface :

- contrainte normale égale à la pression atmosphérique

- à l'amont :

- vitesse et hauteur d'eau imposées

à l'aval :

- condition d'amortissement (milieu poreux)

\subsection{Condition de calcul.}

A l'instant initial, le fluide est au repos, la vitesse et la pression sont imposées comme nulles sur tout le domaine de calcul. Temporellement, on impose en entrée du canal les deux variables suivantes : la vitesse du fluide et la dénivellation de la surface libre correspondant aux caractéristiques d'une houle du deuxième ordre . Les résultats décrits par la suite sont obtenus pour des conditions d'une houle de caractéristiques :

$\cdot \mathrm{T}=1,15 \mathrm{~s}$

- Hauteur crête - creux $=3,010^{-2} \mathrm{~m}$

Différents profils de vitesse sont représentés pour les périodes d'accélération et de décélération, et ce à différentes phases :

\begin{tabular}{|c|c|}
\hline $\begin{array}{c}\text { période d'accélération } \\
\text { (figures 3-a à 3-d) }\end{array}$ & $\begin{array}{c}\text { période de décélération } \\
\text { (figures 3-e à 3-h) }\end{array}$ \\
\hline $180^{\circ} ; 189,8^{\circ} ; 200,0^{\circ} ; 227,2^{\circ} ; 252,2^{\circ}$ & $-3,3^{\circ} ; 5,4^{\circ} ; 13,4^{\circ} ; 39,2^{\circ} ; 64,0^{\circ} ;$ \\
$; 261,8^{\circ} ; 271,4^{\circ} ; 273,3^{\circ} ; 283,0^{\circ} ;$ & $72,7^{\circ} ; 81,6^{\circ} ; 83,4^{\circ} ; 92,7^{\circ} ; 102,3^{\circ} ;$ \\
$292,7^{\circ} ; 318^{\circ} ; 340,8^{\circ} ; 349,2^{\circ}$ et & $130,7^{\circ} ; 159,1^{\circ} ; 169,6^{\circ}$ et $180^{\circ}$ \\
$357,3^{\circ}$ & \\
\hline
\end{tabular}




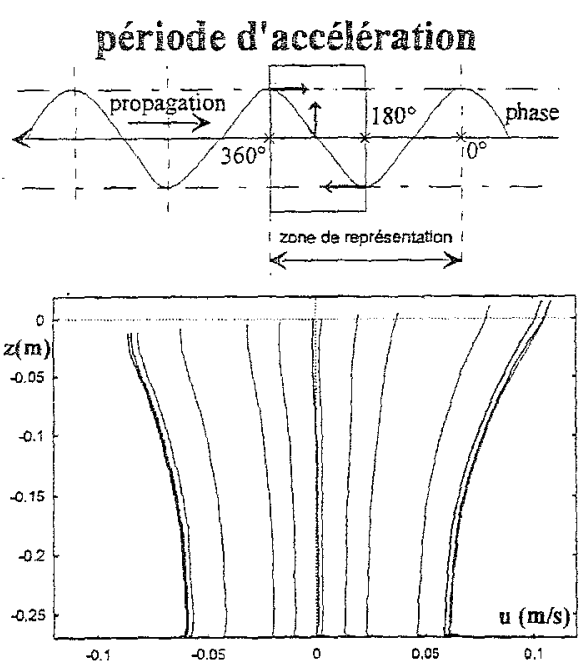

figure 3-a : profil de vitesse horizontale sur toute la hauteur d'écoulement.

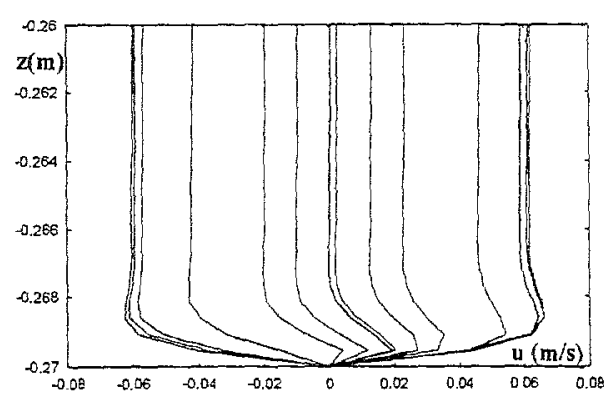

figure 3-b : profil de vitesse au niveau de la couche limite.

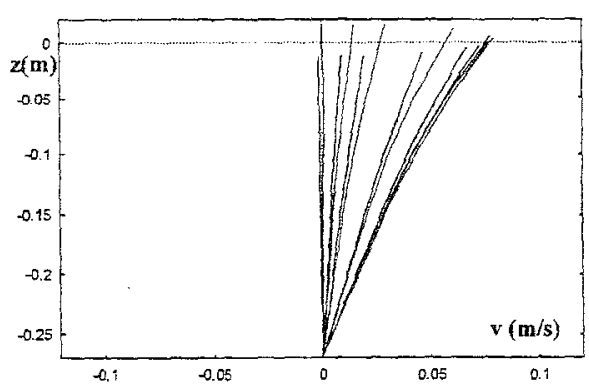

figure 3-c : profil de vitesse verticale sur toute la hauteur d'écoulement.

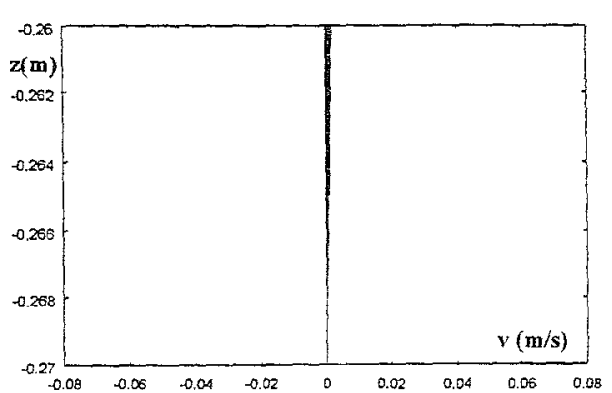

figure 3-d : profil de vitesse verticale au niveau de la couche limite.
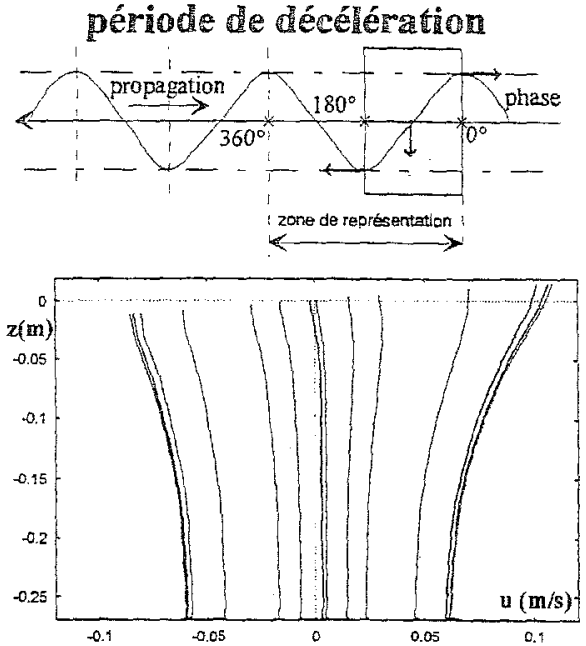

figure 3-e : profil de vitesse horizontale sur toute la hauteur d'écoulement.

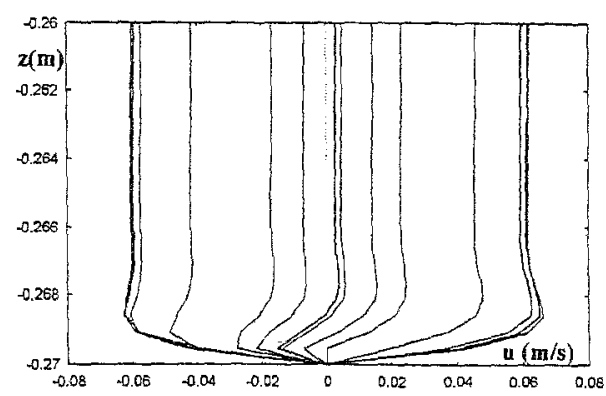

figure 3-f : profil de vitesse au niveau de la couche limite.

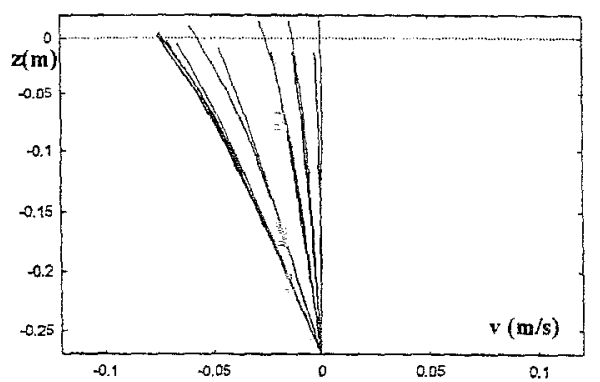

figure 3-g : profil de vitesse verticale sur toute la hauteur d'écoulement.

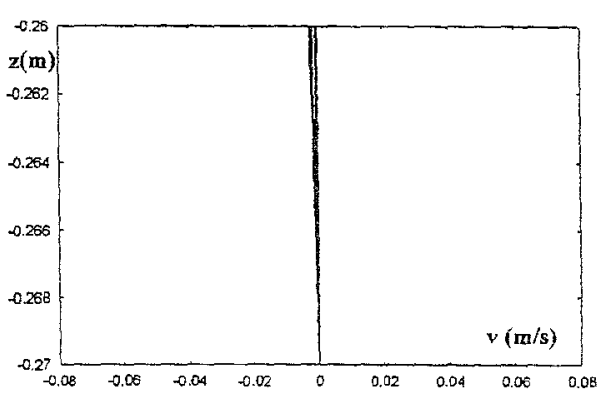

figure 3-h : profil de vitesse verticale au niveau de la couche limite. 


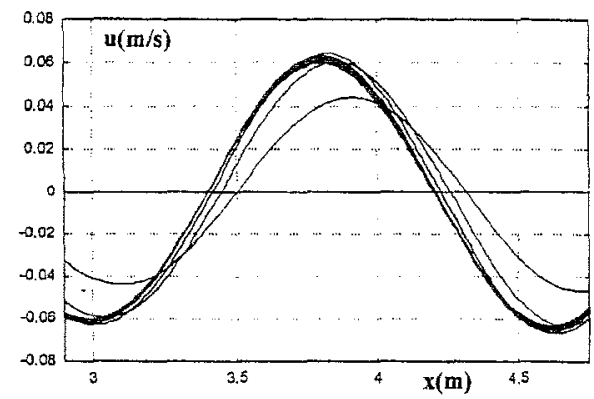

figure 4 : vitesse horizontale pour différentes hauteurs allant du fond à $8 \mathrm{~cm}$ au dessus et ce, sur une longueur d'onde.

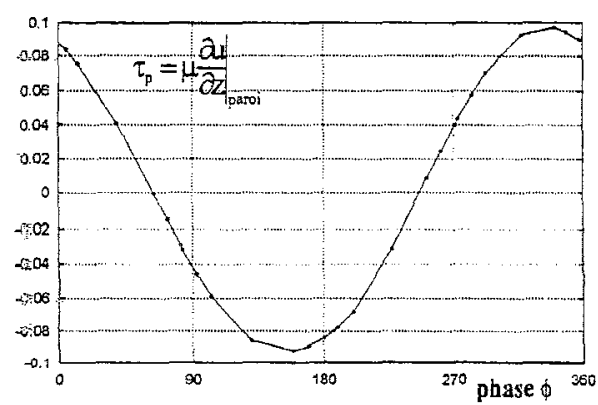

figure 6 : contrainte pariétale sur une période de houle.

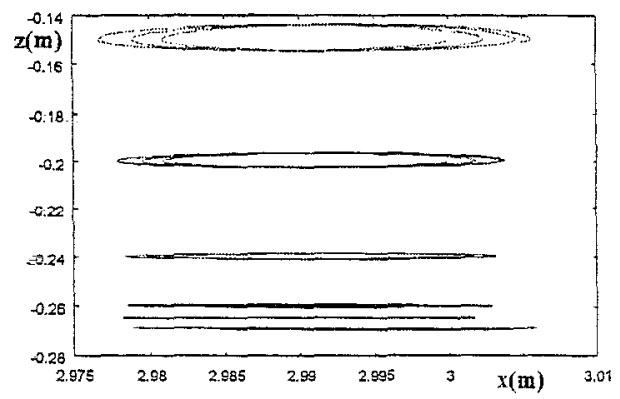

figure 7 : trajectographie spatio-temporelle de plusieurs particules fluides lâchées sur trois périodes à une abscisse initiale de $3 \mathrm{~m}$ et pour différentes hauteurs.

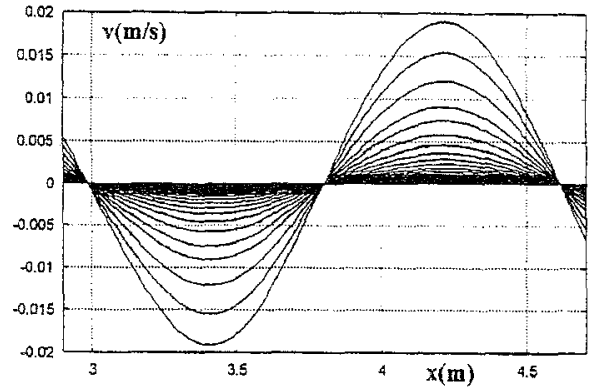

figure 5 : vitesse verticale pour différentes hauteurs allant du fond à $8 \mathrm{~cm}$ au dessus et ce, sur une longueur d'onde.

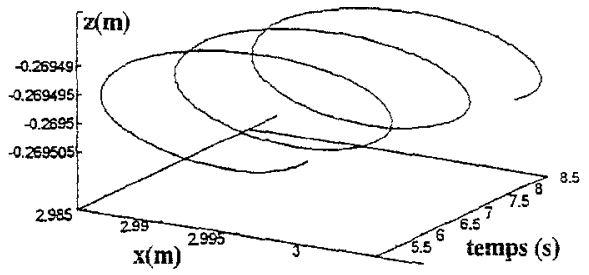

figure 8 : trajectographie d'une particule lâchée à la côte $(3 \mathrm{~m} ;-0,2695 \mathrm{~m})$.

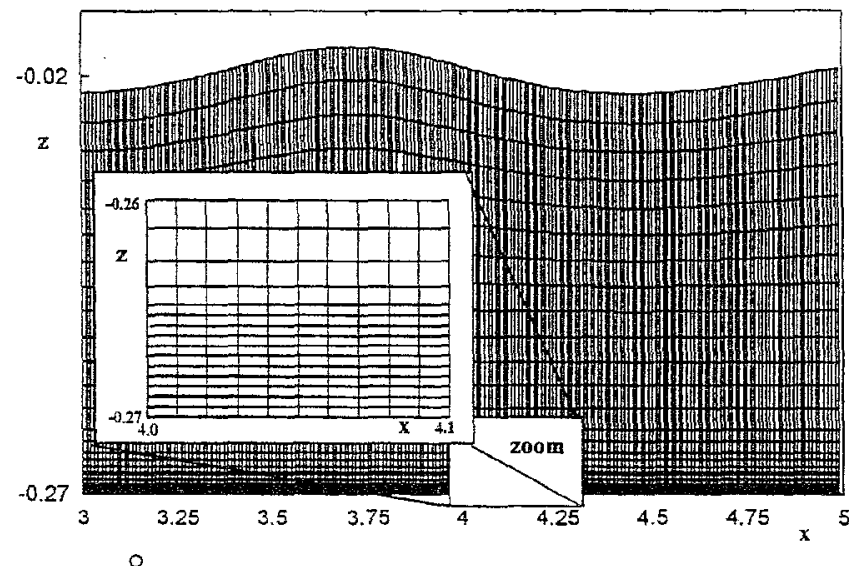

figure 9 : représentation d'une partie du maillage utilisé avec un zoom local près du fond. 


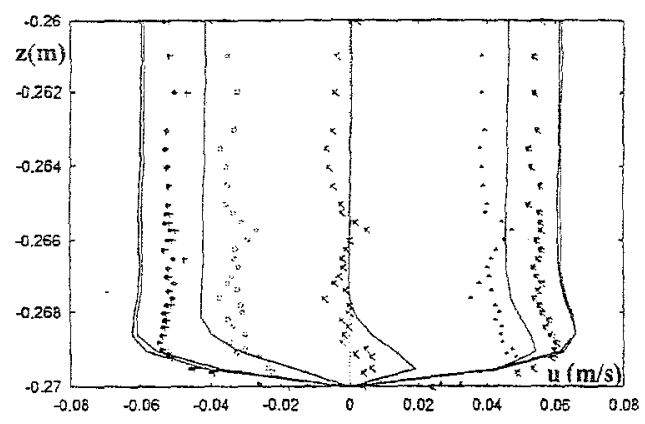

得ure 10 : comparaison des profils de vitesse horizontale obtenus numériquement et expérimentalement sur la phase d'accélération et ce, pour des conditions identiques.

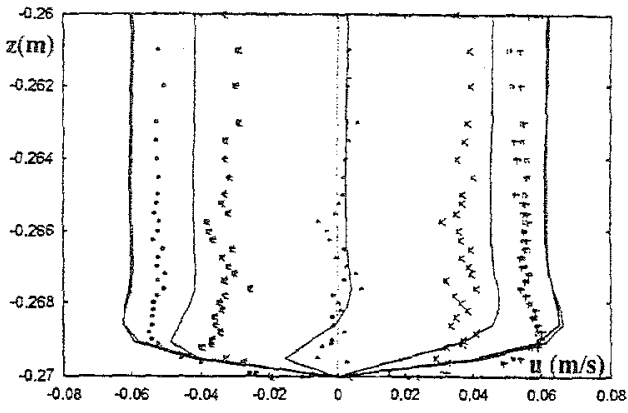

figure 11 : comparaison des profils de vitesse horizontale obtenus numériquement ế expérimentalement sur la phase de décélération et ce, pour des conditions identiques.

Les figures 3b, 3-f et 4 mettent en avant, pour la vitesse, horizontale le déphasage des points appartenant à la couche limite. On retrouve cet aspect pour la contrainte pariétale $\tau_{p}$ (figure 6 ). Ce déphasage apparaît comme inexistant pour les vitesses verticales, figures $3-d, 3-h$ et 5 (l'amplitude de la composante verticale est nulle au niveau de la couche limite).

Une analyse de la trajectographie d'une particule fluide (figures 7 et 8) permet de mettre en évidence une cinématique résiduelle sur toute 1 a hauteur d'eau et ce, dans le sens de propagation de la houle.

Cependant, l'amplitude de la vitesse horizontale au dessus de la couche limite est différente. Deux explications peuvent être envisagées :

- le nombre de points utilisés sur la hauteur de la couche limite est faible (environ 7 points), il ne permet pas d'évaluer correctement la contrainte pariétale $\tau_{p}$ et donc le niveau globale des vitesses.

- expérimentalement, il existe une incertitude sur les mesures faites. La hauteur crête-creux est précise à $+/-2 \mathrm{~mm}$, aussi d'autres calculs ont été effectués (32 et $28 \mathrm{~mm}$ ) et ont permis de montrer une sensibilité appréciable sur le comportement cinématique de la couche limite. De plus, les fluctuations de vitesse englobent les fluctuations réelles de la vitesse ainsi que les erreurs de mesure (pour l'écoulement juste au-dessus de la couche limite, les fluctuations pour la vitesse moyenne horizontale sont de l'ordre de $+/-0,003 \mathrm{~m} \cdot \mathrm{s}^{-1}$ ).

\section{Conclusion.}

Les résultats obtenus par la modélisation numérique sont satisfaisants. Il apparaît néanmoins util d'améliorer la description de la couche limite. Deux solutions ont été envisagées consistant, dans une première étape, à augmenter simplement le nombre de points constituant le domaine discrétisé (on est actuellement limité par la capacité du calculateur utilisé), et, dans une deuxième étape de développer une méthode multi-grilles, multi-domaines et multi-pas de temps permettant d'effectuer un raffinage local du domaine discrétisé là où cela s'avère nécessaire. 